\title{
Study of Mural Paintings Using In Situ XRF, Confocal Synchrotron- $\mu$ - XRF, $\mu$-XRD, Optical Microscopy, and SEM-EDS-The Case of the Frescoes from Misericordia Church of Odemira
}

\author{
S. Valadas, ${ }^{1}$ A. Candeias $,{ }^{1},{ }^{*}$ J. Mirão, ${ }^{2}$ D. Tavares,${ }^{3}$ J. Coroado, ${ }^{4}$ Rolf Simon,${ }^{5}$ A.S. Silva, ${ }^{6}$ M. Gil,${ }^{7}$ A. Guilherme,,${ }^{7}$ and \\ M.L. Carvalho \\ IHERCULES Laboratory and Évora Chemistry Centre, University of Évora and Jose de Figueiredo Conservation Restoration Laboratory, \\ Institute of Museums and Conservation, Largo Marquês de Marialva, 8, 7000-809 Évora, Portugal \\ ${ }_{2}$ HERCULES Laboratory and Geophysics Centre of Évora, University of Évora, Largo Marquês de Marialva, 8, 7000-809 Évora, Portugal \\ 3Alentejo Regional Directorate of Culture, Rua de Burgos 5, 7000-863 Évora, Portugal \\ ${ }_{4}$ Conservation Department, Polytechnic Institute of Tomar, Quinta do Contador, 2300-313 Tomar, Portugal \\ ${ }_{5}$ Forschungszentrum Karlsruhe idHG, ANKA Synchrotron, FLUO Beamline, Karlsruhe, Germany \\ 6Materials Department, National Civil Engineering Laboratory, Av. Brasil 101, 1700-066 Lisbon, Portugal \\ 7Atomic Physics Centre, Lisbon University, Av. Prof. Gama Pinto 2, 1649-003 Lisbon, Portugal
}

\begin{abstract}
In this work, we present the results of an analytical method developed for detailed pigment identification, stratigraphy, and degradation of the paint layers of mural paintings applied in the study of the 17th century frescoes from the Misericordia Church of Odemira (Southwest Portugal). In situ X-ray fluorescence spectrometry analyses were performed on three panels of the mural paintings and complemented by colorimetric measurements. The different color areas were also sampled as microfragments (approx. $1 \mathrm{~mm}^{2}$ ) that were studied as taken or mounted in epoxy resin to expose the different paint layers. The microfragments of paint layers and their cross sections were characterized by optical microscopy and scanning electron microscopy coupled with energy dispersive X-ray spectrometry. Furthermore, elemental analysis was obtained with spatially resolved confocal synchrotron radiation $\mu$-X-ray fluorescence spectrometry performed at ANKA synchrotron FLUO beamline. Occasionally, phase analysis by $\mu$-X-ray diffraction was also performed. Results from the different techniques allowed pigment identification and, in some cases, the evaluation of color changes due to degradation processes and, considering the Southern Portugal geology, the identification of their possible provenance. The pigments used were essentially yellow, brown and red ochres, smalt blue, copper green, and black earths, probably from local sources.
\end{abstract}

Key words: mural paintings, pigments, optical and electron microscopy, in situ XRF, synchrotron micro-XRF and micro-XRD 